\title{
Detection of unrecognized spatial disorientation: A theoretical perspective
}

\author{
Chenru Hao ${ }^{\mathrm{a}, \mathrm{b}}$, Li Cheng ${ }^{\mathrm{a}, \mathrm{b}, *}$, Lisha Guo ${ }^{\mathrm{a}, \mathrm{b}}$, Ruibin Zhao ${ }^{\mathrm{a}, \mathrm{b}}$, Yanru Wu ${ }^{\mathrm{a}, \mathrm{b}}$, Xiuyuan $\mathrm{Li}^{\mathrm{a}}$, \\ Ziqiang $\mathrm{Chi}^{\mathrm{a}}$, Jingjing Zhang ${ }^{\mathrm{a}}$, Xu Liu ${ }^{\mathrm{b}}$, Xiaohan $\mathrm{Ma}^{\mathrm{b}}$, Anqi Wang ${ }^{\mathrm{b}}$, Chunnan Dong ${ }^{\mathrm{b}}$ and \\ Jing $\mathrm{Li}^{\mathrm{b}}$ \\ ${ }^{a}$ Department of Medical Physics, Hebei Medical University, Shijiazhuang, Hebei, China \\ ${ }^{\mathrm{b}}$ Key Laboratory of Medical Imaging Research and Application of Hebei Province, Hebei Medical \\ University, Shijiazhuang, Hebei, China
}

\begin{abstract}
.
BACKGROUND: Spatial disorientation (SD) is a problem that pilots often encounter during a flight. One reason for this problem is that among the three types of SD, there is no validated method to detect the Type I (unrecognized) SD.

OBJECTIVE: In this pursuit, initially we reviewed the problems and the evaluation methods of associated with SD. Subsequently, we discussed the advantages and disadvantages of the subjective questionnaire evaluation method and the behavior evaluation method.

METHODS: On the basis of these analyses, we proposed a method to detect the unrecognized SD that improved the assessment of SD to a significant extent. We developed a new direction to study the unrecognized SD based on the subjective report and the center of pressure $(\mathrm{CoP})$.

RESULTS: The proposed evaluation method can assist the pilots to understand the feelings and physical changes, when exposed to unrecognized SD.

CONCLUSION: We hope that this evaluation method can provide a strong support in developing a countermeasure against the unrecognized SD and fundamentally solve the severe flight accidents arising due to them.
\end{abstract}

Keywords: Aerospace medicine, spatial disorientation, evaluation method, cognition, postural sway

\section{Introduction}

Spatial disorientation (SD) is a problem encountered by many pilots in their flight. SD seriously threatens and affects the flight safety [1]. Gillingham [2] defined SD as a misconception of posture, height, and motion relative to the ground plane. It is accompanied by the pilot's misperception of the gravity vertical line, and misjudgment of the flight attitude relative to other aircraft.

Human beings collect information through the visual system, vestibular system, and proprioception system [3] to determine the orientation and state under normal circumstances. The brain analyzes all kinds of information provided by these systems, and then a person recognizes the correct position. If any

\footnotetext{
${ }^{*}$ Corresponding author: Li Cheng, Department of Medical Physics, Hebei Medical University, Shijiazhuang 050017, Hebei, China; Key Laboratory of Medical Imaging Research and Application of Hebei Province, Hebei Medical University, Shijiazhuang 050017, Hebei, China, E-mail: chengli@ hebmu.edu.cn.
} 
system provides inappropriate or incorrect information, the brain will experience a sensory conflict. The conflict of information provided by these systems can be a cause of SD [4].

Visual factors and vestibular factors are known to cause many SD events. Vision plays a decisive role in the spatial orientation and perception of the surrounding environment. Visual stimulation can easily lead to the wrong estimation of the surrounding environment, such as self-rotation illusion (also known as vection [5]). It is caused by a flowing visual scene, leading to the pilot's wrong judgment of his aircraft's height. When the human body is in a small-angle-tilt state for a long time, it easily leads to the tilt illusion or somato-gravic illusion [1].

There are many symptoms associated with the occurrence of SD. Observers often reported various discomfort symptoms, such as nausea, salivation, light-headedness, dizziness, queasiness, cold sweating, mild restlessness, vertigo, and behavioral disorders [6]. These symptoms are like motion sickness (MS). Although the mechanism of MS is not completely clear, most scholars agree that MS may include conflicts in the sensory perception. Some scholars suggest that MS is caused by SD. Generally speaking, SD and MS have many similarities in their mechanisms and clinical symptoms. Therefore, some references of MS are included in this study.

SD is divided into three types: Type I (unrecognized SD), Type II (recognized SD), and Type III (incapacitating SD). When unrecognized SD happens, the pilots do not feel any discomfort, and do not think that the plane is in an abnormal state. In other words, when unrecognized SD occurs, the pilots experience it, but have no awareness of it. By now, the spatial disorientation is thought to occur, since the pilot does not receive an enough external stimuli to make the pilot aware of the problem in flight. The consequences of this type of SD are often severe. Most type I SD leads to the controlled flight into terrain (CFIT). Although many serious accidents are caused by unrecognized SD, there is a lack of research because unrecognized SD is under the cognitive threshold.

Recognized SD, type II, refers to a kind of misperception in which pilots make false judgments about flight altitude, position, motion. Moreover, they experience conflicts between the actual space state and the instrument's visual space state. Fortunately, pilots get aware of these problems immediately. Incapacitating SD, type III, refers to a kind of mental imbalance, in which pilots misjudge the flight altitude, position, and motion status, and they are aware of these errors. In recent years, scholars agree that the three types of SD are a continuous process [7]. They are divided into three categories based on the different cognitive levels in the brain [3].

Although the research on SD has been carried out for more than four decades, SD still seriously affects the flight safety. $54 \%$ of the flight accidents in the past ten years were related to SD. On an average, Class A accidents occur per million flight hours. With the continuous improvement in the aircraft speed, there is an increase in the accident rate due to SD. Unrecognized SD accounts to 80-85\% of the total accidents arising due to SD, of which a large proportion of them are fatal [8].

Many scholars have researched the SD mechanism, SD measurements, SD countermeasures, and developed training programs for the pilots to avoid SD. Although these researches have outstanding achievements, there are still some problems to be solved. Comparing the aviation accident data in Europe from 1983-1992 and 1993-2002, it can be easily noted that though the total accident rate has decreased from 4.17 times per million flight hours to 2.70 times per million flight hours, the proportion of accidents caused by SD in total accidents has increased from $24.7 \%$ to $33.0 \%$ [9]. These numbers indicate that flight accidents caused by SD are on a persistent increase.

One reason for this problem is that there is a no validated method for the objective measurement for SD. None of the pilots' selection criteria was carried out to screen the pilots' susceptibility to SD. Besides, the most important thing is that although a large number of the existing studies were aimed on the recognized 
SD (type II), only a few studies focus the unrecognized SD that results in the highest proportion of severe flight accidents. Therefore, there is a need to develop an effective method to detect the unrecognized SD. If unrecognized SD can be detected, the countermeasures against it can be definitely implemented. Furthermore, the targeted countermeasures are needed to avoid and mitigate the unrecognized (Type I) SD effects.

In the present study, we summarized the existing SD evaluation methods, and proposed a new method to detect the unrecognized SD, which innovatively completes the identification of unrecognized SD under the cognitive threshold. The developed evaluation method can assist the pilots to understand the feelings and physical changes, when exposed to unrecognized SD. We believe that the proposed evaluation method can provide a strong support for the countermeasure of unrecognized SD and solve the severe flight accidents fundamentally caused by the unrecognized SD.

\section{Assessment of SD}

Considering the research on SD, there is an urgent need for an objective, scientific, and effective evaluation method to study the generation mechanism of SD and develop relevant countermeasures against SD. Scholars have used one or more evaluation methods to assess SD. The evaluation methods include the subjective questionnaires, behavioral methods (eye-movements, flight performance, posture response), neurocognitive methods (electrogastrogram (EEG)) [10], and psychophysiological methods. Physiological data includes: electrogastrography (EGG), electrocardiography (ECG), heart rate (HR), blood pressure (BP), respiratory rate, tidal volume, electromyography (EMG), electrooculogram (EOG), skin electrical activity, and salivary cortisol level. Psychological data includes: questionnaires for analyzing the changes in tension, subjective physical, psychical state, reconstruction interviews, task response time, and accuracy.

The present study focused on developing the subjective questionnaires and posture balance analysis. We devised an objective and scientific detection method suitable for unrecognized SD from the two evaluation methods. Other evaluation methods (EEG signal analysis, physiological parameter detection, psychological parameter analysis, eye movement tracking, flight phenotype, cognitive task completion level, etc.) will be analyzed and dealt with our subsequent studies.

\section{Evaluation using a questionnaire}

Among all the detection methods, the subjective questionnaire is the earliest method used in SD studies. This method plays an essential role in SD detection and assessment. Most of the existing studies have used one or more questionnaires to evaluate SD. The methods include direct questions (questions about the judgment of position and physical state) [11], the questions of symptoms related to SD (vertigo, dizziness, and nausea, etc.) and a questionnaire of a complex scale to confirm the degree and grade of SD.

The use of the questionnaire covers most of the subjective evaluation methods. The content of the questionnaires involves the process of SD, the environment at that moment, actual flight state, pilot's physical condition, and feelings. At present, a series of researches on SD have used the subjective questions or questionnaires to evaluate SD.

\subsection{Pensacola motion sickness questionnaire (MSQ)}

The first questionnaire used to evaluate the orientation problem was the Pensacola motion sickness questionnaire (MSQ) in the 1960s [12,13]. MSQ was based on the scoring scheme designed by Alexander et al. [14]. Since the 1980s, MSQ has been widely used to study the problem of orientation and MS. Later 
in 2001, the motion sickness assessment questionnaire (MSAQ) was modified and processed based on MSQ. Gianaros et al. [15] designed the MSAQ for motion sickness. The results showed that MSAQ was a useful tool to assess motion sickness, and it could be used to evaluate motion sickness from multiple perspectives.

Another questionnaire is the motion sickness susceptibility questionnaire (MSSQ), which assesses the MS caused by an external stimulus [16]. As MSQ is not suitable for mild orientation problems and temporary vertigo, some scholars have designed MSSQ. In a study in 2017, the authors proposed to use the MSSQ-short to evaluate MS susceptibility to mild symptoms, such as visually evoked vection [17]. Vection is also known as relative motion illusion [18]. When participants watched the rotating scene, they will mistakenly think that their bodies were rotating too [19]; this phenomenon is a typical SD.

\subsection{Simulator sickness questionnaire (SSQ)}

Since the 1980s, MSQ has been widely used to study the problem of MS and SD. Some scholars began to design questionnaires for SD and simulator sickness (SS). As stated before, SS, SD, and MS are inseparable [20]. In 1997, Kennedy et al. [21] used SSQ to evaluate the simulator-induced SD. In the follow-up studies by other researchers, SSQ has been applied to evaluate simulator sickness.

In addition to the study of simulator sickness, other scholars had used SSQ to study the effects of exposure to SD in simulated flight [22,23]. Their results indicated that the application of SSQ could provide an assessment evidence for SD. Nevertheless; the existing problems are still very prominent. This questionnaire was not aimed at SD and cannot be merely used to evaluate SD.

\subsection{Spatial disorientation questionnaire}

During the application of SSQ, some scholars designed a special questionnaire for SD. Durnford et al. [24] used a questionnaire for SD in a US Army rotary survey in 1996. The content of this questionnaire was different from the former ones. This questionnaire focused on the frequency of SD episodes, the types of SD, the description, and the classification of SD. The contents of the questionnaire included mild disorientation, significant disorientation, and severe disorientation. The results confirmed that young pilots are more susceptible to SD than older pilots, indicating that this questionnaire played a due role in evaluating SD. Similar results were reported by Wang et al. [25] and Sharon Holmes et al. [26]. They found a significant difference in the score of the scale with or without SD flight experience.

Subsequently, a study on SD by Stephens [3] used a subjective questionnaire confirming that it was feasible to use a questionnaire to evaluate SD.

Some scholars studied vertigo along with the occurrence of spatial disorientation, like Subtil et al. [27]. The questions they used included the specific flight environment as well as the degree and duration of vertigo. Christine M. et al. [28] improved the grading and degree of vertigo based on the previous study. They proved that the subjective questionnaire method was reliable in the SD evaluation.

The latest study was carried out by Helena et al. in 2020 [29]. They designed a detailed SD evaluation questionnaire to assess the effect of SD training. Their result shows that this SD questionnaire could be used to evaluate the training result. However, the questionnaire is not during SD but after the end of the SD experience. Therefore, although this evaluation method is useful, it is not objective.

\subsection{Analysis and summary}

The symptoms of SD are usually similar to those of motion sickness and simulator sickness [30]. According to most scholars' consensus, SD, simulator sickness, and MS belong to the orientation 
disorders, which overlap each other [3]. Therefore, it was reasonable to evaluate SD with MSQ or SSQ. The questionnaire results were straightforward to understand the status of the pilots, especially those in the in-flight training.

Although this method is useful, there are still some problems associated with it. Participants usually fill in the questionnaire before or after the experiment. So, it is not timely and the participants' exact feeling when they are experiencing SD cannot be truly depicted. Therefore, the subjective component is large, which varies with different pilots. Moreover, it is not easy to get a consistent flight environment and flight-feelings encountered during the SD. Therefore, some studies adopt the subjective response to SD without setting too many questions or interrupting the experiment [31].

Moreover, the existing questionnaire method works on the condition that the pilots experienced SD and recognized SD. In these studies, the subjective investigation results of 'no SD' are classified as the non-occurrence of SD. However, according to the SD classification, unrecognized SD can occur, even if there is no subjective feeling of SD. As a result, unrecognized SD cannot be identified solely by the questionnaire method. Therefore, this evaluation method is efficient in Type II (recognized SD) and Type III (incapacitating SD). However, it cannot give an adequate evaluation of the unrecognized SD.

\section{Evaluation by behavior data}

Ethology is the study of human responses to stimuli in internal and external environments. Many studies have shown that during the occurrence of SD, the cognitive ability and flight performance of the pilots decrease significantly. Besides, the behavior of the postural sway is also closely related to the occurrence of SD. Thus, the analysis of postural sway can also be used in the medical field.

The behavioral evaluation method plays an essential role in the countermeasures against SD, including the correctness and efficiency of operation and the behavioral characteristics under flight illusion (eye movement, head movement, body sway, etc.). Human balance and posture are mainly determined from the external information from the visual, vestibular, and proprioceptive systems. The postural response is generally matched with the visual environment. Spatial disorientation may occur, when the visual information conflicts with the vestibular or proprioceptive information, causing a postural instability. When SD occurs, the participants showed an involuntary tilt of the body. In this case, complete vestibular information is essential for the orientation. Therefore, it is vital to stabilize the head and improve the vestibular input for the posture control.

\subsection{Posture control in vection}

Vection is a kind of SD, which is indirectly correlated with MS through the posture activity. The relationship between the postural sway and vection has been reported previously [32]. Since 2002, several postural sway studies have used force platforms to collect the center of pressure (CoP) changes in the experiment [33,34]. They concluded that there were significant differences in the CoP between the vection and non-vection.

Subsequently, in a study in 2015, Da-Silva et al. [35] adopted a dynamic virtual stimulus device to cause vection and analyzed the $\mathrm{CoP}$ data from the participants at different speeds and directions of visual scenes. Their results showed that under relative motion illusion, the human body's posture balance was broken, and the plantar pressure center showed apparent changes.

More and more evidence shows that the postural instability differences can predict vection and SD, when exposed to optical flow stimulation $[32,36]$. No doubt, observing a moving field of vision can 
lead to disorientation [37], which may be reflected in postural adjustments. However, in some visually induced SD conditions, the postural sway differences were usually too small to threaten balance [38]. This condition is known as unrecognized SD, and it is the biggest threat to the safety of the flight. Therefore, even pilots do not cognize SD. It is still possible to find problems from their postural sway.

\subsection{Posture control in $M S$}

In addition to the studies on the postural sway exposed to vection, some scholars used the postural stability to evaluate the susceptibility of MS. According to Riccio and Stoffregen [39], postural instability is an indicator of MS. Some scholars were in an agreement with this view [40,41]. The stability of the body of the participants was an important factor to predict whether they were likely to experience the visually induced motion sickness (VIMS) or not [42].

Laboissiere et al. [43] investigated the relationship between the susceptibility to MS and posture stability. The results showed that the subjects with a history of MS showed more high-frequency components in the visual flow scene. As for visually induced MS, participants with a history of MS exhibited more postural swings in the low-frequency signal. The result concluded that the specific power spectral density (PSD) of the participants assessed the MS levels.

Kim [44] used the virtual reality technology to induce MS. In their research SSQ and posture control were used to evaluate the degree of disorientation in the participants. The results showed that different visual scenes induced different degrees of MS. Moreover, the reduction in motion sickness was related to a stable posture control in the participants, and vice versa. The study suggested that the severity of SD in the participants was directly proportional to the difficulty in the control of the postural balance.

\subsection{Analysis and summary}

Postural sway is usually regarded as an objective index to evaluate the vection. Visually induced postural sway can occur without any subjective illusory [45,46]. Furthermore, the degree of visually induced postural sway has increased along with the rising of visual scene motion. Kuno et al. [47] reported that the increase in the visually induced postural sway resulted in an additional vection. Recent research has even shown that the postural instability can successfully predict vection [48].

The above studies show that the postural sway is closely related to the occurrence of SD. The posture balance is often used in the medical to determine whether the orientation function is normal. The data from postural sway is objective and not affected by the subjective factors. This sway includes two situations: 1) the subjects do not think there is a sway in their body or 2) the subjects report a sway in their body. In the first case, this condition is under the cognitive threshold and belongs to the unrecognized SD. In the second case, this condition is over the cognitive threshold and belongs to the recognized SD. Therefore, the behavioral evaluation method can be applied to evaluate both the unrecognized SD and recognized SD.

\section{Assessment of unrecognized SD}

Based on the analysis and summary of the above research, the present study proposed an evaluation method for the unrecognized SD that was named as the subjective report with postural sway (SRPS). This evaluation method has been tried in our previous research and proved to be feasible [49]. It is hoped that this evaluation method for unrecognized SD can provide a strong support for the screening of the 
pilots and developing countermeasures against the unrecognized SD, thereby solving the serious flight accidents caused fundamentally by the unrecognized SD.

Herein, the advantages and details of the evaluation method are discussed. The evaluation method combines the subjective reports with the CoP data to detect the unrecognized SD. Participants who were exposed to the visual flow were asked to keep their bodies as much as possible still. Then, due to the sensory conflict, SD appears in some participants, leading to a postural sway. Throughout the experiments, the CoP data and the participants' subjective reports (whether they experience SD) were recorded.

\subsection{Subjective report}

The focus of this detection was unrecognized SD, which the participants did not recognize. At the end of each trial, the participants answered whether they experienced SD or not. Here, SD is defined as the feeling that the body was moving or spinning.

The participants' answer fell into two categories:

1) if the participant answered 'Yes, I am moving or spinning', he experienced recognized SD (the illusion of relative motion). Here, ' + ' is used to represent this case.

2) if the participant answered 'No, I am not moving or spinning.', he might experience unrecognized $\mathrm{SD}$ or did not experience SD at all. Here, '-' is used to represent this case.

\subsection{Postural sway (CoP oscillation)}

To verify whether the CoP oscillation of participants significantly deviated on exposure to the visual flow, it was necessary to determine a normal $\mathrm{CoP}$ oscillation range when the participants were not exposed to visual flow. The normal range was obtained from the control trials.

In this experiment, the test time was 30 seconds each time, the sampling frequency was $1000 \mathrm{~Hz}$, the filtering was $100 \mathrm{~Hz}$ low-pass filter and the data of COPX and COPY was collected, using the Bertec FP4060-08 as the force platform.

In the original data collected by the force platform, the origin of the coordinate was located at the center of the force platform. However, the coordinate of the pressure center could be negative in this case. For the convenience of subsequent analysis, we first transformed the origin to the lower left corner of the force platform through the coordinate transformation, so that all coordinate points of the pressure center were positive. Then, a MATLAB program was devised to filter the collected data and used a low-pass Butterworth filter with a cut-off frequency of $10 \mathrm{~Hz}$. Each participant underwent at least six controlled trials (no visual stimulation, balanced standing) before each experiment. The $\mathrm{CoP}$ data of these control trials was calculated to obtain a confidence interval of 95\% [50], which was used as the normal CoP oscillation range.

When the participant was exposed to visual flow, the CoP data was recorded, and the Standard Deviation $(\sigma)$ of $\mathrm{CoP}$ was calculated [51]. The value of $\sigma$ represents the dispersion of the CoP.

The purpose of the deviation calculation was to know whether the subject exhibited SD, while receiving the visual stimulation. As a result, the total standard deviation of $\mathrm{COP}(\sigma)$ in both the $\mathrm{X}$ and $\mathrm{Y}$ axes was used. The formula to calculate the total $\sigma$ in the plane is as follows:

$$
\sigma=\sqrt{\sum\left[\left(x_{m}-x_{i}\right)^{2}+\left(y_{m}-y_{i}\right)^{2}\right] / n}
$$

where, $x_{m}=\sum x_{i} / n, y_{m}=\sum y_{i} / n, x_{i}, y_{i}$ are the coordinates of the $i^{\text {th }}$ sampling point in the $\mathrm{X}$ direction and $\mathrm{Y}$ direction respectively, $n=30000$. 
Table 1

Evaluation method of unrecognized SD

\begin{tabular}{ccl}
\hline Subjective answer & Standard deviation & Result \\
\hline+ & + & Recognized SD \\
+ & - & Recognized SD \\
- & + & Unrecognized SD \\
- & - & Non-SD \\
\hline
\end{tabular}

The standard deviation $(\sigma)$ was used to study whether the subjects exhibited SD during visual stimulation. Each subject was subjected to 12 trials under an experimental stimulus, including 6 control trials and 6 test trials. A confidence interval of $95 \%$ was obtained by using the data of these six control trials, in which the variance was unknown and the confidence interval of the mean was found. The T-statistic was obtained by the expression:

$$
T=\frac{\bar{x}-\mu}{s / \sqrt{n}} \sim t(n-1)
$$

The confidence interval was obtained by:

$$
\left(\bar{x}-\frac{s}{\sqrt{n}} t_{1-\frac{\alpha}{2}}(n-1), \bar{x}+\frac{s}{\sqrt{n}} t_{1-\frac{\alpha}{2}}(n-1)\right)
$$

The formula to calculate $\mathrm{s}$ is as follows:

$$
s^{2}=\frac{1}{n-1}\left(\sum_{i=1}^{n} x_{i}^{2}-n \bar{x}\right)
$$

The $s \bar{x}$ was calculated as:

$$
\bar{x}=\sum_{i=1}^{n} x_{i}
$$

$n=6, \alpha=0.95, t_{1-\frac{\alpha}{2}}(n-1)=t_{0.975}(5) \approx 2.57$.

If the $\sigma$ of $\mathrm{CoP}$ exceeded the maximum confidence interval in the trials of the visual flow, it was judged that the postures sway exhibited a statistically significant deviation. In this case, this deviation was due to SD. On the contrary, when the $\sigma$ value was less than or equal to the maximum of the confidence interval, it was judged that there was no statistically significant deviation in the postures sway. In this case, participants did not experience SD.

In general, if $\sigma$ fell to the right side of the confidence interval, $\mathrm{CoP}$ data of this test was significantly different from that of the control group (represented by + ). If $\sigma$ fell to the left side of the confidence interval, there was no significant difference with the control group (represented by - ).

\subsection{Subjective report with postural sway (SRPS)}

By combining the CoP oscillation with the subjective answer, the detection of unrecognized SD was made (Table 1) as shown as follows:

1) Recognized SD: The subjective answer was 'Yes' $(+)$, and the $\sigma$ was to the right side of the confidence interval $(+)$. The participant knew that he experienced the illusion of relative motion and was not able to control his body quietly.

2) Recognized SD: The subjective answer was 'Yes' $(+)$, and the $\sigma$ was to the left side of the confidence interval (-). The participant knew that he experienced the illusion of relative motion, and he was able to control his body quietly. 
3) Unrecognized SD: The subjective answer was 'No' (-), and the $\sigma$ was to the right side of the confidence interval $(+)$. Visual stimuli affected the posture balance, indicating that the participant was unable to control his body quietly. The participant already experienced a relative motion illusion, but he was unable to cognize it.

4) Non-SD: The subjective answer was 'No' $(-)$, and the $\sigma$ was to the left side of the confidence interval ( $(-)$. The participant did not think that he experienced the illusion of relative motion, and he was able to control his body quietly.

Thus, through the combination of the subjective answer and the objective judgment (CoP oscillation), it was clearly evident whether unrecognized SD occured or not. This method provides a theoretical basis for the detection of the unrecognized SD.

\section{Discussion}

It is a known fact that SD seriously affects the flight safety. Although SD has been studied by many scholars, there is a lack of relevant studies on the unrecognized SD (a type of SD that leads to serious flight accidents). The reason is that unrecognized SD belongs to the subconscious level, which is under the threshold of cognition. Because of the lack of valid evaluation methods for unrecognized SD, the accidents caused by unrecognized SD cannot be solved fundamentally.

In pursuit of these problems, in the present study, we summarized the existing SD evaluation methods. Among these methods, we focused on the advantages and disadvantages of the subjective evaluation method and the behavior evaluation method. Subsequently, we proposed a novel method to detect the unrecognized SD. This method combined the subjective answers with the CoP oscillation and was aimed to detect the unrecognized SD. By adopting this method, it can be realized, whether the pilot experienced the unrecognized SD.

\subsection{Discussion of evaluation methods}

Over the years, many scholars have devoted themselves to assess SD. Moreover, several evaluation methods have been put out for SD, but no effective and uniform evaluation methods exist to evaluate the unrecognized SD.

For subjective assessment, the mainstream method is a questionnaire survey before and after the experiment. It is helpful to evaluate the frequency of SD occurrence and the flight scene. However, his method has some subjective components. It takes much time to fill in the questionnaire, and the pilots' status cannot be recorded in real-time. Therefore, this evaluation method applies to recognized SD above the cognitive threshold [19].

The behavioral assessment is based on an objective evaluation, such as eye-tracking and body posture balance detection. Postural imbalance occurs, when the participant experiences SD. Accordingly, in the trails of no subjective answer of SD, if participants' posture significantly sways, this condition belongs to the unrecognized SD.

\subsection{Advantages of the subjective report with postural sway}

By reviewing and summarizing the existing evaluation methods, we proposed a method that combined the subjective reports with the posture balance to detect unrecognized SD. We named this evaluation method as the subjective report with postural sway (SRPS). The CoP data of participants was collected to 
determine whether SD occurred or not. By analyzing the RMS of CoP, it was objectively determined, whether the participant experienced SD or not.

This evaluation method can provide a theoretical basis to detect the unrecognized SD. In previous studies, we have used this method to detect unrecognized SD and analyzed the EEG signal characteristics, when unrecognized SD occurs [49].

Literature shows that some scholars studied the pilots' cognition and perception of SD using the functional magnetic resonance imaging (fMRI) [7,52]. In addition to fMRI application, positron emission tomography (PET) [53] and magnetoencephalography (MEG) [19] have also been adopted to study SD. Since participants cannot move during fMRI, PET, or MEG scanning, visual flow stimuli was mostly used to induce SD. This pointed out a new direction in the research of unrecognized SD.

\section{Conclusion}

SD is known to seriously affect the flight safety. We put forward an assessment method that combined the subjective reports with the postural sway (SRPS), thus aiming at unrecognized SD. This method confirmed whether the participant was aware of SD and then the behavioral data was analyzed. It makes up for the blank of the detection of unrecognized SD. Using the new evaluation method, we can also help the pilots understand the feelings and physical changes, if they get exposed to unrecognized SD. We hope that this evaluation method can provide a strong support as a countermeasure for unrecognized SD and help to minimize the severe flight accidents fundamentally caused by unrecognized SD.

\section{Acknowledgments}

This work was supported in part by the Bureau of Science and Technology of Hebei Province Key R\&D Program Project (No. 182177170) the Hebei Provincial Foundation for the Introduction of Overseas Personnel (Grant No. C20210344), and the Innovation Experiment Program of College Students in Hebei Medical University (No. USIP2020220, USIP2021029).

\section{Conflict of interest}

None to report.

\section{References}

[1] Newman RL, Rupert AH. The magnitude of the spatial disorientation problem in transport airplanes. Aerosp Med Hum Perf. 2020; 91(2): 65-70. doi: 10.3357/AMHP.5442.2020.

[2] Gillingham KK. A primer of vestibular function, spatial disorientation, and motion sickness. Aeromedical Reviews. 1966; 4: $1-80$.

[3] Stephens MS. Electroneurophysiologic diagnosis of aircraft pilot spatial disorientation. Wright State University, 2003.

[4] David Gradwell DJR. Ernsting's aviation and space medicine 5E.CRC Press, 2016. doi: 10.1136/jrnms-103-147.

[5] Lawson BD. Exploiting the illusion of self-motion (vection) to achieve a feeling of "virtual acceleration" in an immersive display. Proceedings of the 11th international conference on human-computer interaction, Las Vegas, 2005, pp. 1-10.

[6] Kennedy RS, Lane NE, Berbaum KS, Lilienthal MG. Simulator sickness questionnaire: An enhanced method for quantifying simulator sickness. The International journal of aviation psychology. 1993; 3(3): 203-20. doi: 10.1207/s15327108ijap0303_3. 
[7] Hao C, Fan X, Dong C, Qiao L, Li X, Li X et al. A classification method for unrecognized spatial disorientation based on perceptual process. Ieee Access. 2020; 8: 140654-60. doi: 10.1109/ACCESS.2020.3012821.

[8] Holmes SR, Bunting A, Brown DL, Hiatt KL, Braithwaite MG, Harrigan MJ. Survey of spatial disorientation in military pilots and navigators. Aviat Space Environ Med. 2003; 74(9): 957-65.

[9] Klyde DH, Lampton AK, Schulze PC. Development of spatial disorientation demonstration scenarios for commercial pilot training. AIAA Modeling and Simulation Technologies Conference, Washington, DC, USA, 2016.

[10] Hao C, Zhao R, Qiao L, Li X, Zhang J, Wu Yet al. EEG analysis of visually induced spatial disorientation. In 2019 12th International Congress on Image and Signal Processing, BioMedical Engineering and Informatics (CISP-BMEI), 2019, pp. $1-5$.

[11] Owen N, Leadbetter AG, Yardley L. Relationship between postural control and motion sickness in healthy subjects. Brain Res Bull. 1998; 47(5): 471-4. doi: 10.1016/s0361-9230(98)00101-4.

[12] Kelldgg RS, Kennedy RS, Graybiel A. Motion sickness symptomatology of labyrinthine defective and normal subjectives during zero gravity maneuvers. Techn docum rep no. AMRL-TDR-64-47. AMRL-TR. Aerospace Medical Research Laboratories (U.S.). 1964: pp. 1-11.

[13] Kennedy RS, Tolhurst GC, Graybiel A. The effects of visual deprivation on adaptation to a rotating environment. NSAM-918. Research report. Naval School of Aviation Medicine (U.S.). 1965: pp. 1-36.

[14] Alexander SJ, Cotzin M. Studies of motion sickness; The effects upon sickness rates of waves of various frequencies but identical acceleration. Journal of Experimental Psychology. 1947; 37(5): 440. doi: 10.1037/h0063240.

[15] Gianaros PJ, Muth ER, Mordkoff JT, Levine ME, Stern RM. A questionnaire for the assessment of the multiple dimensions of motion sickness. Aviation, Space, and Environmental Medicine. 2001; 72(2): 115-9.

[16] Kennedy RS, Dunlap WP, Fowlkes JE. Prediction of motion sickness susceptibility. Boca Raton, FL: CRC Press, 1990.

[17] Hwang AD, Deng H, Gao Z, Peli E. Quantifying visually induced motion sickness (VIMS) during stereoscopic 3D viewing using temporal VIMS rating. Journal of Imaging Science \& Technology. 2017; 61(6): 1-9. doi: 10.2352/J.ImagingSci.Technol.2017.61.6.060405.

[18] Lubeck AJA, Bos JE, Stins JF. Equally moved and not really sick from viewing 2D and 3D motion stimuli on a TV screen. Displays. 2016; 41: 9-15. doi: 10.1016/j.displa.2015.10.004.

[19] Palmisano S, Allison RS, Schira MM, Barry RJ. Future challenges for vection research: Definitions, functional significance, measures, and neural bases. Front Psychol. 2015; 6. doi: 10.3389/fpsyg.2015.00193.

[20] Kennedy RS, Fowlkes JE, Berbaum KS, Lilienthal MG. Use of motion sickness history questionnaire for prediction of simulator sickness. Aviation Space \& Environmental Medicine. 1992; 63(7): 588-93. doi: 10.1016/0960-1686(92)90423-I.

[21] Kennedy RS, Berbaum KS, Lilienthal MG. Disorientation and postural ataxia following flight simulation. Aviation Space \& Environmental Medicine. 1997; 68(1): 13-7. doi: 10.1001/archfami.6.1.79.

[22] Lewkowicz R, Stróżak P, Bałaj B, Francuz P. Auditory verbal working memory load effects on a Simulator-Induced spatial disorientation event. Aerosp Med Hum Perf. 2019; 90(6): 531-9. doi: 10.3357/AMHP.5277.2019.

[23] Lewkowicz R, Stróżak P, Bałaj B, Francuz P, Augustynowicz P. Selective auditory attention and spatial disorientation cues effect on flight performance. Aerosp Med Hum Perf. 2018; 89(11): 976-84. doi: 10.3357/AMHP.5153.2018.

[24] Durnford SJ, DeRoche SL, Harper JP, Trudeau LA. Spatial disorientation: A survey of U.S. Army Rotary-Wing aircrew. USAARL report no. 96-16. 1996.

[25] Wang J, Li S, Lin P. A psychophysical and questionnaire investigation on the spatial disorientation triggered by cockpit layout and design. Int J Ind Ergonom. 2019; 72: 347-53. doi: 10.1016/j.ergon.2019.06.008.

[26] Holmes SR, Bunting A, Bostock S, Brown L, Hiatt K. Preliminary survey of spatial disorientation in UK military pilots and navigators. The RTO HFM Symposium on "Spatial Disorientation in Military Vehicles: Causes, Consequences and Cures", La Coruña, Spain, 2002.

[27] Subtil J, Varandas J, Galrão F, Dos Santos A. Alternobaric vertigo: Prevalence in Portuguese Air Force pilots. Acta Oto-Laryngol. 2007; 127(8): 843-6. doi: 10.1080/00016480601075415.

[28] Talker CM, Kallus KW. Anticipatorily controlled Top-Down processes influence the impact of coriolis effects. International Symposium on Aviation Psychology, Dayton, 2015.

[29] Pennings HJM, Oprins EAPB, Wittenberg H, Houben MMJ, Groen EL. Spatial disorientation survey among military pilots. Aerosp Med Hum Perf. 2020; 91(1): 4-10. doi: 10.3357/AMHP.5446.2020.

[30] Kallus KW, Tropper K. Evaluation of a spatial disorientation simulator training for jet pilots. International journal of applied aviation studies. 2004; 4(1): 41-56.

[31] Chuang S, Chuang C, Yu Y, King J, Lin C. EEG alpha and gamma modulators mediate motion Sickness-Related spectral responses. Int J Neural Syst. 2016; 26(02): 1650007. doi: 10.1142/S0129065716500076.

[32] Palmisano S, Arcioni B, Stapley PJ. Predicting vection and visually induced motion sickness based on spontaneous postural activity. Exp Brain Res. 2018; 236(1): 315-29. doi: 10.1007/s00221-017-5130-1.

[33] Fushiki H, Kobayashi K, Asai M, Watanabe Y. Influence of visually induced self-motion on postural stability. Acta Oto-Laryngol. 2005; 125(1): 60-4. doi: 10.1080/00016480410015794. 
[34] Thurrell AEI, Bronstein AM. Vection increases the magnitude and accuracy of visually evoked postural responses. Exp Brain Res. 2002; 147(4): 558-60. doi: 10.1007/s00221-002-1296-1.

[35] Da Silva PJG, Cagy M, Infantosi AFC. A new dynamic virtual stimulation protocol to induce vection. 6th European Conference of the International Federation for Medical and Biological Engineering, Springer, Cham, 2015, pp. 497-500.

[36] Apthorp D, Nagle F, Palmisano S. Chaos in balance: Non-linear measures of postural control predict individual variations in visual illusions of motion. Plos One. 2014; 9(12): e113897. doi: 10.1371/journal.pone.0113897.

[37] Barrett GV, Thornton CL, Cabe PA. Cue conflict related to perceptual style. J Appl Psychol. 1970; 54(3): 258-64. doi: 10.1037/h0029079.

[38] Guerraz M, Thilo KV, Bronstein AM, Gresty MA. Influence of action and expectation on visual control of posture. Brain Research. Cognitive Brain Research. 2001; 11(2): 259-66. doi: 10.1016/s0926-6410(00)00080-x.

[39] Riccio GE, Stoffregen TA. An ecological Theory of Motion Sickness and Postural Instability. Ecol Psychol. 1991; 3(3): 195-240. doi: 10.1207/s15326969eco0303_2.

[40] Stoffregen TA, Smart LJJ. Postural instability precedes motion sickness. Brain Res Bull. 1998; 47(5): 437-48. doi: 10.1016/s0361-9230(98)00102-6.

[41] Bonnet CT, Faugloire E, Riley MA, Bardy BG, Stoffregen TA. Motion sickness preceded by unstable displacements of the center of pressure. Hum Movement Sci. 2006; 25(6): 800-20. doi: 10.1016/j.humov.2006.03.001.

[42] Yu Y, Chung HC, Hemingway L, Stoffregen TA. Standing body sway in women with and without morning sickness in pregnancy. Gait Posture. 2013; 37(1): 103-7. doi: 10.1016/j.gaitpost.2012.06.021.

[43] Laboissière R, Letievant J, Ionescu E, Barraud P, Mazzuca M, Cian C. Relationship between spectral characteristics of spontaneous postural sway and motion sickness susceptibility. Plos One. 2015; 10(12): e144466. doi: 10.1371/journal.pone.0144466.

[44] Kim N, Kim B. The effect of retinal eccentricity on visually induced motion sickness and postural control. Applied Sciences. 2019; 9(9): 1919. doi: 10.3390/app9091919.

[45] Previc FH, Mullen TJ. A comparison of the latencies of visually induced postural change and self-motion perception. Journal of Vestibular Research: Equilibrium \& Orientation. 1990; 1(3): 317-23.

[46] Berthoz A, Lacour M, Soechting JF, Vidal PP. The role of vision in the control of posture during linear motion. Prog Brain Res. 1979; 50: 197-209. doi: 10.1016/S0079-6123(08)60820-1.

[47] Kuno S, Kawakita T, Kawakami O, Miyake Y, Watanabe S. Postural adjustment response to depth direction moving patterns produced by virtual reality graphics. The Japanese Journal of Physiology. 1999; 49(5): 417-24. doi: 10.2170/jjphysiol.49.417.

[48] Palmisano S, Apthorp D, Seno T, Stapley PJ. Spontaneous postural sway predicts the strength of smooth vection. Exp Brain Res. 2014; 232(4): 1185-91. doi: 10.1007/s00221-014-3835-y.

[49] Li Y, Chen Y, Lv X, Yang Y, Li J, Hao C et al. EEG functional network properties related to visually induced unrecognized spatial disorientation. Bio-Med Mater Eng. 2015; 26(s1): S1115-24. doi: 10.3233/BME-151408.

[50] Ehrenfried T, Guerraz M, Thilo KV, Yardley L, Gresty MA. Posture and mental task performance when viewing a moving visual field. Cognitive Brain Research. 2003; 17(1): 140-53. doi: 10.1016/S0926-6410(03)00088-0.

[51] Fujino Y, Amimoto K, Fukata K, Inoue M, Shida K, Takahashi H et al. Test-retest reliability of the postural vertical in healthy participants. Progress in Rehabilitation Medicine. 2017; 2: n/a. doi: 10.2490/prm.20170013.

[52] Julian JB, Keinath AT, Marchette SA, Epstein RA. The neurocognitive basis of spatial reorientation. Current biology: CB. 2018; 28(17): R1059-73. doi: 10.1016/j.cub.2018.04.057.

[53] Aurora SK, Barrodale PM, Tipton RL, Khodavirdi A. Brainstem dysfunction in chronic migraine as evidenced by neurophysiological and positron emission tomography studies. Headache. 2007; 47(7): 996-1003, 1004-7. doi: 10.1111/j.15264610.2007.00853.x. 